\title{
Cuidado em saúde à criança na atenção primária em região de fronteira ${ }^{a}$
}

\author{
Child health care in primary care in a border region
} Atención de salud infantil en atención primaria en una región fronteriza

\author{
Taigra Morgana Picco ${ }^{1}$ (C) \\ Maria Aparecida Baggio ${ }^{2}$ (D) \\ Aline Renata Hirano ${ }^{1}$ (D) \\ Sebastião Caldeira ${ }^{2}$ (1) \\ Rosangela Aparecida Pimenta Ferrari ${ }^{3}$ [D \\ 1. Universidade Estadual do Oeste do Paraná. \\ Foz do Iguaçu, PR, Brasil.
}

2. Universidade Estadual do Oeste do Paraná. Cascavel, PR, Brasil.

3. Universidade Estadual de Londrina. Londrina, PR, Brasil.

\begin{abstract}
REsUMO
Objetivo: identificar o cuidado em saúde à criança de zero a 24 meses na atenção primária em uma região de fronteira. Método: estudo qualitativo realizado na Atenção Primária à Saúde, com 14 mães e cinco enfermeiras, com o uso de entrevistas semiestruturadas, de março de 2018 a junho de 2019. A análise temática de conteúdo orientou a análise dos dados. Resultados: identificaram-se visitas domiciliares escassas no seguimento da criança cujos problemas comuns da infância condicionam o acesso aos serviços de saúde para a consulta médica, o uso de práticas populares ou a indicação de medicações por farmacêuticos/ atendentes de farmácia; o acesso às Unidades de Pronto Atendimento em detrimento das unidades de Atenção Primária por carência de pediatras neste nível de atenção, além de carência de especialistas e morosidade para a realização de exames na rede de saúde. Conclusão: a carência de profissionais de saúde, de especialistas na rede e de recursos materiais são obstáculos a serem superados para o cuidado da criança em região de fronteira. O seguimento da criança brasileira residente no Paraguai requer planejamento.
\end{abstract}

Palavras-chave: Atenção primária à saúde; Cuidados de Enfermagem; Enfermagem; Saúde da criança; Saúde na fronteira.

\section{Abstract}

Objective: to identify the health care provided to children from zero to 24 months of age in primary care in a border region. Method: a qualitative study conducted in Primary Health Care, with 14 mothers and five nurses, with the use of semi-structured interviews, from March 2018 to June 2019. Thematic content analysis guided the data analysis. Results: scarce home visits were identified in the follow-up of children whose common childhood problems condition access to health services for medical consultation, the use of popular practices or the indication of medications by pharmacists/pharmacy assistants; access to Emergency Care Units instead of Primary Care units due to the lack of pediatricians in this level of care, in addition to the lack of specialists and slowness to perform exams in the health network. Conclusion: the lack of health professionals, of specialists in the network, and of material resources are obstacles to be overcome for the care of children in the border region. The follow-up of Brazilian children living in Paraguay requires planning.

Keywords: Border health; Child health; Nursing; Nursing care; Primary health care

\section{REsumen}

Objetivo: identificar la atención de salud para niños de cero a 24 meses en atención primaria en una región fronteriza. Método: estudio cualitativo realizado en Atención Primaria de Salud, con 14 madres y cinco enfermeras, mediante entrevistas semiestructuradas, de marzo de 2018 a junio de 2019. El análisis de contenido temático guió el análisis de datos. Resultados: se identificaron escasas visitas domiciliarias en el seguimiento de los niños cuyos problemas comunes de la infancia afectan el acceso a los servicios de salud para la consulta médica, el uso de prácticas populares o la indicación de medicamentos por parte de los farmacéuticos / asistentes de farmacia; el acceso a las Unidades de Urgencias en detrimento de las Unidades de Atención Primaria por la falta de pediatras en este nivel de atención, además de la falta de especialistas y retrasos en la realización de pruebas en la red sanitaria. Conclusión: la falta de profesionales de la salud, especialistas en la red y de recursos materiales son obstáculos a superar para el cuidado del niño en la región fronteriza. El seguimiento de los niños brasileños que residen en Paraguay requiere planificación.

Palabras clave: Atención primaria de salud; Cuidado de Enfermería; Enfermería; Salud infantil; Salud fronteriza.
Autor correspondente:

Aline Renata Hirano

E-mail: alinerenatahirano@gmail.com

Recebido em 16/04/2021.

Aprovado em 15/08/2021.

DOI:https://doi.org/10.1590/2177-9465-EAN-2021-0104 


\section{INTRODUÇÃO}

A saúde da criança apresenta-se como campo prioritário de ações no âmbito do Sistema Único de Saúde (SUS), uma vez que a mortalidade infantil representa um índice internacional acerca das condições de saúde e qualidade de vida da população'.

A Organização Mundial de Saúde (OMS) preconiza que o número de óbitos infantis dos municípios, Estados e países seja inferior a dois dígitos a cada mil Nascidos Vivos (NV). No Brasil, em 2018, foram contabilizados 12,35 óbitos a cada mil NV. Ao levar em consideração o mesmo índice e período de avaliação, o Estado do Paraná notificou 10,3 mortes e Foz do Iguaçu, município na região leste do Estado, fronteira com a Argentina e Paraguai, teve o registro de 10,8 casos $^{2,3}$. Como descrito, apesar da significativa redução da mortalidade infantil no país nas últimas décadas, observa-se, ainda, que os números atuais divulgados pelos órgãos responsáveis estão aquém do que se considera aceitável pela OMS.

Entre os anos de 1990 e 2015, registrou-se queda de cerca de $68 \%$ no número de mortes em crianças menores de cinco anos. Contudo, em 2015, 90\% dos casos registrados foram de crianças menores de um ano. A prematuridade tem se mantido como a principal causa de morte ao longo das últimas duas décadas, permanecendo a diarreia, as infecções respiratórias e a desnutrição entre as nove primeiras colocações ${ }^{4}$.

Os dados nacionais observados entre os anos de 1990 e 2015 são bastante otimistas, no entanto, demonstram a fragilidade quanto ao cuidado necessário frente às causas evitáveis e sensíveis de acompanhamento pela Atenção Primária à Saúde (APS) em gestantes e, principalmente, em crianças menores de dois anos de idade 4 .

Os fatores associados à causa de diarreia e a problemas respiratórios em crianças menores de dois anos em Bangladesh, com a participação de mais de 3500 crianças, foram a depressão materna e a insegurança alimentar e nutricional das famílias, apontando para a importância de programas e políticas públicas que incluam ações voltadas a essa temática ${ }^{5}$.

De acordo com o Fundo das Nações Unidas para a Infância (UNICEF), as doenças associadas à desnutrição e diarreia podem ser evitadas com condições apropriadas de saneamento básico e serviço de saúde pública adequado no cuidado infantil ${ }^{1}$. Desse modo, a disseminação de informações sobre a prevenção e minimização de complicações das doenças é importante para a diminuição de agravos ${ }^{6}$.

No ano de 1993, a OMS e a UNICEF elaboraram a estratégia denominada de Atenção Integrada às Doenças Prevalentes na Infância (AIDPI) com o objetivo de reduzir a mortalidade associada às doenças prevalentes na infância de crianças menores de cinco anos ${ }^{6}$. Contudo, para que essa iniciativa seja efetiva, é importante que os profissionais de saúde estejam aptos a tal prática e que recebam suporte para atender a população infantil de acordo com o que é preconizado pela $\mathrm{OMS}^{7}$.

De acordo com uma das diretrizes da Política Nacional de Atenção Integral à Saúde da criança (PNAISC), as ações voltadas à saúde infantil devem ser organizadas levando em consideração as Redes de Atenção à Saúde (RAS) ${ }^{8}$. Estas configuram o modelo de atenção à saúde atual no âmbito do SUS, cujo objetivo é oferecer, por meio dos serviços de saúde, um cuidado integral e contínuo que atenda aos princípios e às diretrizes desse sistema de saúde. Dentre as ações propostas, estão as voltadas à imunização, às visitas domiciliares, à promoção da amamentação e alimentação saudável, à continuação do cuidado, entre outras, cujo objetivo está além da sobrevivência e do crescimento da criança, priorizando a vigilância e a promoção da saúde infantil para o seu pleno desenvolvimento biopsicossocial ${ }^{9}$.

Foz do Iguaçu, contexto desta pesquisa, está localizada na região oeste do Estado do Paraná, na fronteira com o Paraguai e a Argentina. No Brasil, existem peculiaridades na assistência à saúde na fronteira quando se compara às demais regiões. Dentre elas, está o deslocamento frequente da população residente nos países vizinhos em busca de atenção à saúde em detrimento de condições melhores de acesso e cuidado ${ }^{10}$.

Os residentes nos países vizinhos, particularmente no Paraguai, buscam assistência em território brasileiro, em especial, para a atenção maternoinfantil. Mulheres paraguaias ou brasileiras que vivem no Paraguai acessam o serviço de saúde brasileiro para o nascimento de seus filhos. Estas crianças, de nacionalidade brasileira, têm direito ao atendimento pelo SUS. No período de 2006 a 2012, dos 34.456 nascidos no Brasil, 95,7\% são de mulheres residentes no Brasil e 4,3\%, de mulheres residentes no Paraguai ${ }^{11}$.

Diante do exposto, questiona-se: como se dá o cuidado em saúde à criança de zero a 24 meses na atenção primária em uma região de fronteira? O objetivo do estudo foi identificar o cuidado em saúde à criança de zero a 24 meses na atenção primária em uma região de fronteira.

\section{MÉTODO}

Estudo qualitativo, realizado na APS, com 14 mães de crianças de zero a 24 meses de idade e cinco enfermeiras, guiado pelas recomendações do Consolidated Criteria for Reporting Qualitative Research (COREQ).

Foram critérios de inclusão: ser mãe de criança de zero a 24 meses de idade, usuária do sistema público de saúde, em realização de puericultura na APS; ser enfermeiro atuante diretamente na prestação de cuidado à criança na APS na realização de puericultura. Foram incluídos enfermeiros e mães de cada um dos cinco distritos sanitários do município de Foz do Iguaçu, Paraná, Brasil. Foram excluídas do estudo mães que não são responsáveis pelo cuidado integral da criança e enfermeiras que estavam afastadas do trabalho no período da coleta de dados.

A coleta dos dados foi realizada na APS, em Unidades Básicas de Saúde (UBS), tanto do modelo tradicional (conhecidas como "unidades abertas") quanto da Estratégia Saúde da Família (ESF), uma de cada distrito sanitário do município estudado, que está localizado na região oeste do Estado do Paraná, na fronteira do Brasil com o Paraguai e a Argentina e, segundo o Instituto Brasileiro de Geografia e Estatística (IBGE), possui uma 
população estimada, em 2017, em 263.915 habitantes, formada por uma diversidade de etnias, com 82 nacionalidades distintas.

A seleção das participantes foi por conveniência. $O$ convite às mães foi realizado na ocasião das consultas de puericultura. A coleta de dados foi guiada por roteiro semiestruturado, validado por teste-piloto e aplicado por uma mestranda, no período de março de 2018 a junho de 2019, iniciado pela questão norteadora: "Comente sobre o cuidado da criança na atenção primária após o nascimento".

As entrevistas foram realizadas nas próprias unidades de saúde, de forma individual, com duração de aproximadamente 30 minutos, e registradas em dispositivo eletrônico de áudio. Com as mães, aconteceram antes ou após as consultas de puericultura das crianças e, com as enfermeiras, conforme a disponibilidade delas, nas unidades onde atuavam. As transcrições das entrevistas retornaram aos participantes para conferência, mas somente duas enfermeiras retornaram, contudo, sem modificações.

A análise temática de conteúdo orientou a análise dos dados. Na primeira etapa, de pré-análise, realizou-se a leitura flutuante e interpretativa dos dados, seguida de leitura exaustiva para a definição das unidades temáticas conforme o objetivo do estudo. Na segunda etapa, de exploração dos dados, foi realizada a operação de codificação por meio de recortes do texto em unidades de registros ou de significados. Nesta etapa, também foram realizadas a classificação e a agregação das informações das unidades de registro, que conduziram a formação das categorias temáticas. Na terceira etapa, foi realizado o tratamento dos resultados por meio de inferência e interpretação dos dados das categorias temáticas ${ }^{12}$.

Trata-se de recorte de uma pesquisa multicêntrica intitulada: "Rede Mãe Paranaense na perspectiva da usuária: o cuidado da mulher no pré-natal, parto, puerpério e da criança", a qual foi aprovada pelo Comitê de Ética em Pesquisa com Seres Humanos da Universidade Estadual de Londrina com o CAAE número 67574517.1.1001.523. Para a garantia do anonimato, as participantes foram identificadas por letras que as representam seguidas da ordem de participação no estudo: "E" para enfermeira e "M" para mãe. Exemplo: E1...E5, M1...M14.

\section{RESULTADOS}

\section{Caracterização dos participantes}

As mães apresentaram idades entre 18 e 44 anos, cujas crianças possuíam a faixa etária entre um ano e quatro meses a dois anos de idade, em sua maioria, brasileiras (com exceção de uma haitiana e uma paraguaia), casadas ou vivendo em união estável, com Ensino Médio completo, exercendo profissão ou ocupação, com renda familiar média entre um e três saláriosmínimos e residindo com mais de três pessoas no domicílio, tendo uma delas moradia no Paraguai.

Em relação aos profissionais de saúde, eram todos compostos por enfermeiras, com idades entre 32 e 38 anos, atuando há mais de dez na APS, todas com especialização, sendo uma em nível de mestrado.

\section{Cuidado em saúde e práticas populares à criança}

Esta categoria refere-se ao cuidado em saúde às crianças nas condições e doenças comuns da infância, identificadas em consultas de puericultura pelas enfermeiras e/ou relatadas pelas mães durante o atendimento na APS, à forma de manejo do profissional frente a elas, bem como às práticas de cuidado com a criança realizadas pelas mães e baseadas em crenças familiares. As principais condições ou doenças relatadas são: alergias; assaduras; doenças respiratórias; candidíase oral e vaginal; cólicas; desidratação; diarreia e vômitos; doenças gripais febres; icterícia neonatal; infecções gastrointestinais; infecção urinária; conjuntivites; micose de pele; piolho; verminoses etc.

Para estas condições, as crianças receberam consulta médica e/ou pediátrica com a indicação de tratamento ou orientações aos pais conforme cada caso. Contudo, sobre as orientações profissionais recebidas, nota-se a insegurança das mães para executar cuidados básicos, como a limpeza nasal, necessários para a melhora da condição clínica da criança.

[...] nessa unidade, tem muita micose de pele, geralmente, a mãe passa para o bebê [...], assadura, [...] porque utilizam muito lenço umedecido por ser prático. É bem comum a candidíase oral, monilíase oral e vaginal também [...] na parte da vulva da criança [...]. Eu mais vejo [...] desidratação, as alergias de pele, conhecida como grosseirões [...] casos de febre, gripes, doenças pulmonares, recém-nascidos com icterícia [...], infecção urinária [...], vômitos e diarreias, principalmente quando as crianças começam com leite diferente, as fórmulas ou de caixinha. (E1)

$[. .$.$] tosse seca [. .$.$] só me passaram um xarope [...] o$ médico me receitou [...] faço em casa a inalação [...]. O médico, ele disse para fazer em casa aquela lavagem no nariz dela [...]. Eu nunca fiz a lavagem nela porque eu tenho medo de fazer isso [...]. (M5)

Teve gripe, cólica, teve dor de barriga [...] ela parou de tomar o meu leite [...] eu não tinha dinheiro em casa e misturei o leite de caixinha com água para dar para ela [...] ela teve dor de barriga [...]. (M8)

Após o nascimento, o aparecimento de cólicas no recémnascido é a queixa mais comum, que comumente condiciona o acesso das mães à unidade de saúde. Além de uso de medicações prescritas pelos médicos para minimizá-las, nota-se o uso de práticas populares como a oferta de chás, a realização de massagens com óleos e plantas, a aplicação de calor e outros comumente recomendados por familiares e pessoas próximas.

[... ela chorava muito de dor, a enfermeira me ensinou a fazer exercícios com as perninhas dela, massagem, e 
isso dava uma aliviada. Como ela tinha muita cólica, a médica me passou um remedinho. (M3)

Começou a cólica, daí, minha mãe faz o negócio com a arruda e o óleo, pode ser com arruda e óleo quentinho ou com alho e óleo, passa a mão nas costelinhas e vai puxando assim na barriguinha, fazendo massagem bem de leve; no começo, eu colocava a faixinha, mas depois eu pararei de colocar e colocava só um pano, uma fralda. Eu passo o ferro na fralda, deixo bem quentinha, daí, eu só enrolo, não aperto igual a faixinha, só enrolo ela assim, daí, ela dorme bem. (M6)

[...] minha vó, ela sempre deu chá de camomila pra gente [...] ela indicou e eu dei pra ele, mas, para cólica mesmo, eu dava o Simeticona ou Colikids [...]. (M12)

Os profissionais de saúde não orientam o uso de chás. Entretanto, conforme os relatos das enfermeiras, essa e outras práticas fazem parte do cuidado pelas mães para o alívio das cólicas e a promoção de conforto às crianças.

[...] não recomenda e nem orienta o consumo de chá. Mas elas acabam dando escondido por causa da cólica que a criança apresentou ou por causa que a vizinha, a irmã ou a mãe falou [...]. (E2)

[...] tem muita mãe que dá o chá, o medicamento e também faz algo para fazer massagem, como usar óleo quente com um pouco de arruda para passar na barriga da criança. Procuro saber porque, em algum momento, pode ser o único recurso que a mãe teve, naquele momento, para ajudar o filho. Assim, eu não gosto, mas compreendo as diferentes situações. Tem mãe que dá só porque a amiga deu e a criança não tem nada [...]. (E5)

Acessar uma farmácia e solicitar ao farmacêutico, ou mesmo ao atendente de farmácia, a indicação de medicações para as condições comuns da infância (doenças ou seus sinais e sintomas) é uma prática comum das mães confirmada por enfermeiras. Logo, as medicações são administradas às crianças sem a prévia avaliação médica. Contudo, quando o quadro da criança tem piora e requer tratamento com o uso de antibióticos, as mães recorrem à APS para a consulta médica.

[...] vou direto e compro na farmácia. É mais fácil, tipo Dipirona, Paracetamol [...]. (M1)

Medicamentos eu sempre compro na farmácia, nunca usei o do posto. Na verdade, a gente automedica ele. Vou sempre na mesma farmácia. Eu já conheço as pessoas [...] tenho confiança neles [...] compro só com o mesmo farmacêutico [...]. (M12)

[...] se a criança ficou doente, é bem comum elas [mães] irem na farmácia por conta própria buscar os medicamentos. Elas só procuram o médico para poder comprarmedicamento para crianças quando elas precisam de antibiótico porque a farmácia não vende. Elas buscam o posto para poder conseguir a receita médica. (E4)

\section{Referência em saúde à criança}

Segundo as enfermeiras, as crianças são referenciadas para serviços de outros níveis de complexidade quando não conseguem ter resolutividade na APS, como ao serviço de atenção especializada, quando requerem investigação por especialista, ao serviço de atenção hospitalar, no caso do neonato, ou à Unidade de Pronto Atendimento (UPA), nas condições de urgência ou emergência infantil.

Nessas condições, o transporte é realizado por meio do Serviço de Atendimento Móvel de Urgência (SAMU). A referência entre os serviços da rede de saúde é realizada por meio de telefonema e de encaminhamento escrito pela equipe de Enfermagem ou médica, acompanhado de um detalhamento do diagnóstico da criança, exame físico e motivo do encaminhamento.

[...] quando a criança está muito desidratada, por um quadro de diarreia mais grave, que não consegue mais hidratação via oral, a gente encaminha para UPA ou no caso de amarelão, como as mãe falam, a gente encaminha direto para o Costa, que é o hospital referência até os 28 dias após o nascimento da criança; eu faço uma referência por escrito, faço um exame físico, avaliando que essa criança estava ictérica, como ela estava esclerótica, se está urinando pouco, a cor da urina; nesse caso, eu já chamo a médica para vir avaliar na minha sala e a gente encaminha ela para o hospital. (E1)

Sobre a referência de crianças para a atenção secundária ou especializada, as enfermeiras referem, como principais fragilidades, a insuficiência de especialistas na RAS e a demora para a autorização e a realização de exames de alto custo. Elas apontam a necessidade de maior disponibilidade de médicos especialistas, fonoaudiólogos, psicólogos e outros profissionais na rede municipal - no Núcleo Ampliado de Saúde da Família (NASF).

[...] quando essa criança precisa de uma especialidade, o nosso município já encontra algumas dificuldades [...] não é uma dificuldade da unidade básica, é uma dificuldade da rede [...]. Já as especialidades são poucos profissionais para atender, liberação de exames de alto custo [...] a gente vai continuar tentando solucionar o problema aqui, porém, o recurso, a gente não tem [...]. (E2)

[...] falta outros profissionais na área [...]. Isso não é luxo. Isso é o básico. Precisamos de mais profissionais no NASF para atender à demanda da população e desafogar um pouco as equipes das unidades para trabalhar em conjunto. (E1) 
Importa salientar que, quando as crianças são referendadas ao serviço especializado, as mães mantêm as consultas de puericultura na APS. Assim, a continuidade do cuidado da criança permanece nos dois níveis de atenção.

[...] eu vou no cardiopediatra por causa do problema do coração dele e continuo vindo aqui, no posto de saúde, para realizar as consultas com a Fulana [médica] e também para acompanhar o Ciclano [criança] na puericultura dele [...]. (M1)

[...] essa criança sempre é acompanhada aqui. Ela é mais acompanhada aqui por ela estar mais próximo da casa dela [...]. A gente tem o suporte do agente de saúde [...], tem a consulta agendada com o pediatra, mas, mesmo assim [...], a gente acaba sabendo mais da rotina dela e da família do que os especialistas [...]. A gente conhece a mãe, os irmãos, a casa, a situação que a criança está, a condição que ela está vivendo [...]. (E1)

\section{Fragilidades da APS: obstáculos e estratégias para contorná-las}

Identifica-se que as visitas domiciliares pelos profissionais de saúde, até o $5^{\circ}$ dia de vida das crianças, após a alta hospitalar, não aconteceram para a totalidade das crianças da área de cobertura das unidades de saúde. Segundo os enfermeiros, a falta de carro para uso pelos profissionais da APS dificulta a realização de visitas domiciliares. Salientam preferir que as mães acessem a unidade para os cuidados de Enfermagem.

[...] eu não recebi visita não, ninguém foi lá, olha, que eu nem saí de casa, fiquei o tempo todo por causa do resguardo. É igual eu falei, eu não recebi visita não. (M3)

[...] eu não recebi visita não depois que ela nasceu [...]. (M13)

[...] a gente não tem como fazer as visitas domiciliares para todas as mães; prefiro que essa mãe venha no posto, então, eu faço antes do décimo dia. Na verdade, eu acho que é inviável, para mim, marcar e realizar essa consulta na casa da paciente [...] dependo de um carro que não fica aqui na unidade; o carro, ele é dividido entre seis equipes, então, querendo ou não, aqui, eu tenho mais instrumento para poder ajudar [...]. (E1)

[...] prefiro que elas venham aqui [...] fui algumas vezes nas casas, mas chegava lá e as mães não queriam que entrássemos na casa delas [...]. (E2)

A visita domiciliar é realizada na forma de busca ativa às mães que não compareceram à UBS, na primeira semana de vida da criança, para iniciar a puericultura. Frequentemente, são os Agentes Comunitários de Saúde (ACS) que realizam a busca ativa orientados pelas enfermeiras.
[...] eu faço visita domiciliar [...] não compareceu na unidade, tipo, deu sétimo e o oitavo dia, aí, a gente faz a busca ativa na casa, mas a maioria das pacientes eu vejo no posto [...]. (E1)

Geralmente, o ACS que fazem a busca ativa dessas mães em suas residências [...]. (E5)

Foram identificadas barreiras para a continuidade do cuidado de crianças de mães brasiguaias ou paraguaias, nascidas em território brasileiro, visto que o estudo foi realizado em região de fronteira. Essas crianças, por terem naturalidade brasileira, têm direito de acesso à saúde no país. Contudo, após o nascimento, as mães não comparecem às unidades de saúde APS, no Brasil, para o acompanhamento da criança por meio da puericultura.

Convém reportar que as mães/mulheres brasiguaias buscam atendimento de pré-natal no Brasil, em serviço específico para acompanhamento de gestantes/crianças brasiguaias, com a vinculação do nascimento de suas crianças em território brasileiro para a garantia de acesso à saúde a elas. Já as paraguaias - não residentes no Brasil - recorrem ao serviço de saúde brasileiro para o nascimento de seus filhos com a mesma garantia. Além disso, algumas conseguem o atendimento por meio do uso de endereços de amigos ou parentes residentes no país para obter o cartão do Sistema Único de Saúde (SUS). Logo, a busca ativa das crianças faltosas na puericultura é impossibilitada, visto que a criança reside com a mãe no Paraguai.

[...] ACS não consegue acompanhar porque elas vêm específico para consulta, elas dão endereço de algum parente, algum amigo. Essa mulher mora no Paraguai [...]. Só que não tem como a gente negar o atendimento porque ela tem o direito. [...] então, elas já têm o cartão SUS. [...] a maioria delas não são paraguaias, elas têm documento brasileiro, só que elas moram lá no Paraguai [...]. São brasileiras e acabaram casando com paraguaios. [...] elas têm totalmente o direito [de acesso à saúde] no Brasil e, nesse caso, elas são as brasiguaias. [...] paraguaias, quando a criança nasce no Brasil, automaticamente, essa criança tem total direito, é o que a maioria está fazendo, vindo ganhar seus filhos aqui e depois tem acesso à saúde [...]. Você não vai ficar vendo se ela mora mesmo no Paraguai ou não porque, querendo ou não, essa não é a nossa função. (E1)

As mães revelaram diferentes situações experienciadas para o acesso em saúde à criança. Nota-se que a APS não é a primeira porta de acesso para todas as mães que precisam de atendimento às suas crianças. Quando buscam acesso neste nível de atenção, nem sempre encontram pediatra para atender à demanda requerida. A UPA, por sua vez, absorve as demandas da atenção primária por falta de pediatra neste nível de atenção e mostra-se ser escolha frequente pelas famílias, para acesso em saúde, em busca de resolutividade. Logo, a falta de profissionais da APS gera sobrecarga às UPAs. 
[...] eu prefiro ir direto para UPA porque aí chega no posto e não tem médico ou o médico vai demorar para atender, uma, que eles atendem criança mais rápido. (M8)

[...] as UPAs sempre cheias, querendo ou não, quando não conseguimos resolver na unidade, sobrecarrega os demais sistemas de saúde, tipo a UPA [...]. (E4)

Neste sentido, a falta de profissionais na APS configura fragilidade para a garantia da continuidade do cuidado das crianças, sendo necessário prover profissionais em quantitativo adequado para atender à demanda da população atendida.

[...] pouca gente para 'trabalhá', pouco médico, enfermeira, as meninas, na vacina ali, sempre corrido e cheio [...]. (M6)

[...] eu acho que é pouco gente trabalhando, a população só cresce, sempre vai ter mais gente por eles atenderem, então, eles têm que colocar mais profissionais, mais pessoas para trabalhar, senão eles não vão conseguir. (M9)

Entende-se que a insuficiência de profissionais de saúde, de materiais e de medicamentos na APS, bem como a manutenção e a qualidade dos materiais existentes, configura um problema de saúde pública, indicando a necessidade de os gestores em saúde proverem essas deficiências para o atendimento adequado e qualificado às crianças. Assim, para que haja o aperfeiçoamento da continuidade do cuidado da criança no município de Foz do Iguaçu, torna-se necessário o fortalecimento da APS, em estrutura e ações, com empenho pelos gestores e, por conseguinte, pelos profissionais de saúde. Consequentemente, a APS será mais bem valorizada pela população.

[...] essa falta de funcionários nas equipes é um problema da rede, no contexto geral, muitas unidades estão sem profissional para acompanhar essas crianças, tanto no acolhimento quanto na puericultura, é um problema geral, um problema de política, teria que entrar alguém que olhasse realmente com olhar para atenção básica, a solução tá aqui embaixo, já passou da hora de achar que é só um médico e enfermeira que são essenciais dentro da unidade, a população enxerga o postinho como algo simples e o hospital como algo grande, algo complexo [...] enquanto a gente não valorizar a importância de uma régua antropométrica para realizar as medidas, não vai resolver [...]. (E1)

[...] eu fui na farmácia para comprar os remédios [...], no posto, falta muito remédio. Tipo antibiótico não tem, daí, o médico prescreve e vamos na farmácia comprar. (M9)

[...] existe sala de vacina fechada por falta de profissional ou problema na geladeira, problema de manutenção, carro para as visitas domiciliares, materiais educativos, a gente precisava de uma régua antropométrica e veio uma régua de isopor, muito fraquinha, a gente acabou preferindo ficar com uma velha de ferro. Outra coisa: o pedido de material feito de baixa qualidade, a farmácia também que poderia ter aqui [...]. (E1)

[...] já tive uma vez que estava faltando vacina, tive que encaminhar a mãe para outra unidade [...] a geladeira acabou estragando e, infelizmente, algumas mães ficaram com o calendário vacinal atrasado [...]. (E3)

O agendamento mensal e o encaixe de consultas de puericultura, quando a mãe faltou ao horário agendado, são ações inerentes à prática profissional do enfermeiro, consideradas estratégias pelos enfermeiros, valorizadas pelas mães, que podem assegurar o cuidado continuado da criança.

[... ] a enfermeira já deixa tudo agendado para a próxima vez; como já fica tudo agendado, acho que é até mais fácil assim, a consulta de puericultura para outra vez. (M2)

[...] um dia que estava chovendo muito, eu não consegui vim [...] ficou uns dois dias chovendo direto, daí, quando parou, eu vim no posto, eu disse que estava chovendo muito e não vim naquele dia [...] a enfermeira pediu para 'esperá' um pouquinho que ela estava atendendo uma mulher, daí, depois, ela ia me 'atendê'. Ela foi, me 'chamô' e ela foi 'fazê' a consulta da nenê. (M4)

[...] na verdade, a gente, da abertura na agenda, realiza um encaixe de casos específicos; eu sei que aquela mãe vem a cada dois meses, daí, ela veio na realização de uma consulta médica, já realizamos tudo junto: consulta médica e puericultura [...] demora mais, demora, mas agiliza o processo. (E3)

Os dados em questão revelaram as dificuldades e as fragilidades no contexto da APS, com evidente comprometimento da continuidade do cuidado da criança residente em região de fronteira, particularmente de crianças nascidas no Brasil, mas residentes em países vizinhos. Além disso, a APS não se mostra consolidada como porta de entrada para o atendimento de crianças por precariedade da oferta dos serviços de profissionais como pediatra e enfermeiro em tempo integral.

\section{DISCUSSÃO}

No Brasil, as doenças mais comuns da infância, na faixa etária entre zero e cinco anos de idade (desnutrição, anemia e as infecções respiratórias), possuem agravos que, apesar de poderem ser controlados no nível primário de atenção à saúde, ainda permanecem entre as dez causas mais comuns de mortalidade infantil, sendo que $90 \%$ das mortes que acometeram crianças em 2015 foram de menores de um ano. Essas e outras doenças foram confirmadas neste estudo, contudo, na faixa etária de até dois anos - idade das crianças das quais as mães participaram da pesquisa4.

Quanto aos episódios de cólicas no recém-nascido, além do uso de medicamentos e métodos farmacológicos, os pais relataram 
realizar ações para aliviar os sinais de dor, como massagens com ervas e óleos quentes e a oferta de chás medicinais. Contudo, as práticas de saúde consideradas complementares ou alternativas nem sempre apresentam comprovação científica ou, por vezes, são objetos de estudos considerados de baixa qualidade devido à presença de vieses que acabam por reduzir a indicação de seu uso por profissionais de saúde ${ }^{13}$.

Um estudo realizado na Romênia revelou que $70 \%$ dos pais participantes da pesquisa automedicavam os filhos, sendo que $60 \%$ destes apresentavam interesse na homeopatia e/ou fitoterápicos, geralmente utilizados a partir de sua experiência, e $37 \%$ deles buscavam informações junto ao farmacêutico, prática observada nesta pesquisa ${ }^{14}$.

Diante dessa realidade, em especial por tratar-se de uma região de fronteira como a que se configura nesta pesquisa, em que, como citado anteriormente, convivem cerca de 82 nacionalidades diferentes, torna-se essencial que o profissional de saúde, as políticas públicas locais e a forma de organização do sistema de saúde considerem as práticas culturais presentes nesse contexto. Para isso, devem ser fortalecidas as habilidades e atitudes no atendimento ao usuário, pautadas na competência cultural em que se preze pela valorização e aceitação das vivências e dos costumes da pessoa atendida, reconhecendo as possibilidades de inclusão desse modo de cuidado no processo saúde-doença, construindo, junto do usuário, a melhor forma de atenção à saúde, contemplando as questões biológicas, sociais e culturais, favorecendo, assim, o cuidado integral, uma vez que a simetria entre o diálogo usuário-profissional é importante para o sucesso terapêutico ${ }^{15,16}$

No cenário delineado, as famílias que, em tese, deveriam ser atendidas na APS encontram respostas mais imediatas na UPA. Essa condição deve-se à carência de profissionais na APS para atendimento às crianças, considerada um agravante ao acesso que gera, como consequência, sobrecarga à atenção especializada. Isso define uma rede com pactuações e fluxo frágeis e sobrecarregados, na qual as UPAs ocupam demandas que seriam mais bem acolhidas na APS ${ }^{17}$.

Além disso, as barreiras encontradas nos serviços de atenção primária, na realização do encaminhamento às especialidades (por carência de profissionais especialistas), tornam o atendimento pouco resolutivo e incapaz de propor a continuidade do cuidado desejado à criança nos serviços de saúde ${ }^{18}$.

Essa situação é reflexo de uma Rede Atenção à Saúde deficiente, o que demonstra a necessidade do estabelecimento de um fluxo de atendimento em que os canais de comunicação e as ferramentas de referência e contrarreferência entre os profissionais de todos os níveis de atenção ocorram de forma integrada na perspectiva do cuidado centrado na criança ${ }^{18}$.

$\mathrm{Na}$ APS, a realização das visitas domiciliares é prevista pela Rede Mãe Paranaense (RMP) até o 5ํ dia de vida após a alta hospitalar, devendo ocorrer o comparecimento da criança na unidade de saúde até o $10^{\circ}$ dia de vida da criança para o início da puericultura, visto que esse é um período de grande vulnerabilidade ao recém-nascido ${ }^{19}$. Contudo, os dados apontam que os enfermeiros não costumam realizar as visitas domiciliares para todas as mães no período recomendado conforme a RMP e a busca ativa realizada pelo ACS nem sempre acontece de forma efetiva.

Essa dificuldade está vinculada à falta de meio de transporte para a locomoção e de profissionais na unidade de saúde para atender às demandas existentes. Essas situações inviabilizam que a estratégia AIDPI seja realizada de forma efetiva na APS, uma vez que, além da importância de capacitação dos profissionais quanto à sua utilização na prática de cuidado infantil, existe a necessidade de recursos, como os citados anteriormente, para a sua implementação satisfatória ${ }^{20}$.

Quanto à busca ativa, esta torna-se impossibilitada quando se trata de crianças de famílias brasiguaias (brasileiros residentes no Paraguai) ou estrangeiras que utilizam endereço de parentes ou amigos para conseguir o atendimento no sistema público de saúde brasileiro, quando, na realidade, residem no país vizinho. A fim de assegurar o acesso ao SUS, um dos principais arranjos por famílias estrangeiras residentes fora do Brasil é a utilização de comprovantes residenciais, muitas vezes, pelo "empréstimo" de endereços adquiridos por meio da cumplicidade de brasileiros ou, ainda, pelo aluguel de casas no Brasil ${ }^{21,22}$.

É importante salientar que é por meio da visita domiciliar que as enfermeiras e toda a equipe de saúde da APS podem conhecer o contexto em que as famílias vivem, construir vínculo com elas e orientá-las quanto ao cuidado necessário à saúde da criança ${ }^{23}$.

No Brasil, a região de fronteira possui peculiaridades quando comparada às demais regiões em relação à assistência à saúde. Um dos motivos está relacionado à procura de atendimentos por estrangeiros residentes nos países vizinhos, portanto, de forma inapropriada, vinculada à avaliação positiva destes em relação à assistência à saúde ofertada pelo SUS comparada à dos demais países fronteiriços ${ }^{10}$.

Foz do Iguaçu, município pertencente à tríplice fronteira, contempla essas características, apresentando particularidades na prestação da continuidade do cuidado maternoinfantil. As unidades centrais que atendem à demanda da população flutuante (brasileiros residentes no Paraguai), por vezes, apresentam dificuldade na realização da continuidade do cuidado infantil, já que muitas famílias buscam atendimento apenas em casos associados a alguma comorbidade da criança e não realizam o acompanhamento por meio da puericultura.

Essa dificuldade na continuidade do cuidado das crianças brasileiras residentes no Paraguai provoca, além da falta de assistência, que pode não existir no país vizinho, prejuízos ao diagnóstico da situação em saúde infantil no Brasil, tanto em relação à atenção à saúde das crianças quanto às condições de saúde destas ${ }^{24}$.

Neste caso, a desigualdade regional, geográfica e social existente no Brasil, de modo singular, neste estudo, desenvolvido em uma região de fronteira, representa uma fragilidade que configura um problema de saúde pública. Estrangeiros buscam assistência em território brasileiro, particularmente para a atenção 
maternoinfantil, que afeta a oferta e o financiamento de serviços do país. Por exemplo, mulheres paraguaias ou brasileiras que vivem no Paraguai acessam o serviço de saúde brasileiro para o nascimento de seus filhos. Logo, estas crianças, de nacionalidade brasileira, têm direito ao atendimento pelo sistema de saúde brasileiro, alertando sobre a importância de ações governamentais por meio de políticas públicas que considerem tal situação característica dessa região ${ }^{25}$.

Isso pode ser confirmado no estudo, já apresentado anteriormente, que mostrou que $4,3 \%$ dos nascimentos no Brasil, entre os anos de 2006 e 2012, foram de mulheres que declararam residir no Paraguai. Contudo, após o nascimento, as mães brasiguaias ou paraguaias nem sempre realizam a puericultura de forma efetiva e frequente ${ }^{11}$. Convém destacar que essa situação é agravada, uma vez que não há acordos internacionais que viabilizem a continuidade do cuidado da criança por profissionais de saúde do Brasil na zona de fronteira entre os diferentes países.

Apesar das fragilidades identificadas, o estudo apresenta estratégias adotadas pelas enfermeiras para a promoção da continuidade do cuidado da criança na APS, entre elas: educação em saúde; estímulo materno; agendamento, encaixes e remanejamento, quando necessários, das consultas mensais de puericultura. Quanto às consultas de puericultura, acolher e atender as mães em suas necessidades repercutem em satisfação, apesar dos obstáculos inerentes à APS ${ }^{26}$, condição confirmada neste estudo.

\section{CONCLUSÃO}

As doenças comuns na infância presentes na literatura também foram observadas neste estudo, assim como a prática de medicalização das crianças por parte dos pais, sem orientação médica, e o uso de tratamentos alternativos e complementares como os chás medicinais.

O atendimento infantil no contexto do estudo acontece, em sua maioria, na APS, contudo, pela falta de profissionais, em especial, do médico pediatra, e pela demora do atendimento, as crianças também são atendidas nas UPAs. No entanto, a criança em atendimento na APS, quando reportada para o serviço especializado, permanece acompanhada na APS, um aspecto favorável para a garantia da continuidade do cuidado da criança.

As fragilidades nas ações e serviços da APS relacionam-se à falta de profissionais de saúde e de recursos que inviabilizam as práticas fundamentais no cuidado à criança, como a visita domiciliar. A oferta de especialistas é requerida para atender à demanda referenciada pela APS. E, ainda, a continuidade do cuidado à criança brasileira residente no Paraguai é prejudicada devido à ausência nas puericulturas e à impossibilidade de busca ativa no país vizinho. Assim, o seguimento da criança brasileira residente no Paraguai requer planejamento.

O desenho do estudo limitou-se a entrevistar mães e enfermeiros da APS. Para ampliar a investigação acerca do tema, recomenda-se estudo com a participação de outros profissionais de saúde.

\section{FINANCIAMENTO}

Edital Universal/CNPq no 01/2016, Processo n‥ 407508/20163 , concedido à pesquisa com o título: Rede Mãe Paranaense na perspectiva da usuária: o cuidado da mulher no pré-natal, parto, puerpério e da criança, coordenado por Rosangela Aparecida Pimenta Ferrari.

\section{CONTRIBUIÇÕES DOS AUTORES}

Desenho do estudo: Taigra Morgana Picco. Maria Aparecida Baggio.

Coleta ou produção dos dados: Taigra Morgana Picco.

Análise de dados: Taigra Morgana Picco. Maria Aparecida Baggio. Aline Renata Hirano. Sebastião Caldeira. Rosangela Aparecida Pimenta Ferrari.

Interpretação dos resultados: Taigra Morgana Picco. Maria Aparecida Baggio. Aline Renata Hirano. Sebastião Caldeira Rosangela Aparecida Pimenta Ferrari.

Redação e revisão crítica do manuscrito: Taigra Morgana Picco. Maria Aparecida Baggio. Aline Renata Hirano. Sebastião Caldeira. Rosangela Aparecida Pimenta Ferrari.

Aprovação da versão final do artigo. Taigra MorganaPicco. Maria Aparecida Baggio. Aline Renata Hirano. Sebastião Caldeira. Rosangela Aparecida Pimenta Ferrari.

Responsabilidade por todos os aspectos do conteúdo e a integridade do artigo publicado: Taigra Morgana Picco. Maria Aparecida Baggio. Aline Renata Hirano. Sebastião Caldeira. Rosângela Aparecida Pimenta Ferrari.

\section{EDITOR ASSOCIADO}

Aline Cristiane Cavachilli Okido (D)

\section{EDITOR CIENTÍFICO}

Ivone Evangelista Cabral (D)

\section{REFERÊNCIAS}

1. The United Nations Children's Fund. Levels \& Trends in Child Mortality [Internet]. UNICEF; 2019 [citado 2020 ago 15]. Disponível em: https:// data.unicef.org/resources/levels-and-trends-in-child-mortality/

2. Instituto Brasileiro de Geografia e Estatística. Tábuas completas de mortalidade no Brasil [Internet]. 2019 [citado 2020 ago 15]. Disponível em: https://www.ibge.gov.br/estatisticas/sociais/populacao/9126-tabuascompletas-de-mortalidade. $h t m l ?=\& \mathrm{t}=0$-que-e.

3. Secretaria de Estado da Saúde do Paraná (PR). Superintendência de Vigilância em Saúde - SVS. Centro de Epidemiologia - CEPI. Divisão de Informações Epidemiológicas -DVIEP. Mortalidade infantil [Internet] 2018 [citado 2018 nov 2] Disponível em: http://www.saude.pr.gov.br/ arquivos/File/Mortalida_Materna_e_Infantil_18_07_2018.pdf

4. França EB, Lansky S, Rego MAS, Malta DC, França JS, Teixeira R et al. Leading causes of child mortality in Brazil, in 1990 and 2015: estimates from the Global Burden of Disease study. Rev Bras Epidemiol. 2017;20(20, suppl. 1):46-60. http://dx.doi.org/10.5123/S1679-49742019000100006. PMid:28658372.

5. Ullah MB, Mridha MK, Arnold CD, Matias SL, Khan AS, Siddiqui Z et al. Factors associated with diarrhea and acute respiratory infection in children under two years of age in rural Bangladesh. BMC Pediatr. 
2019;19(386):386. http://dx.doi.org/10.1186/s12887-019-1738-6. PMid:31656181.

6. Ministério da Saúde (BR). Secretaria de Atenção à Saúde, Departamento de Ações Programáticas e Estratégicas. Manual AIDPI neonatal [Internet] Brasília: Ministério da Saúde; 2014 [citado 2020 mar 27]. Disponível em: http://portalarquivos.saude.gov.br/images/pdf/2016/fevereiro/03/ Manual-Aidpi-corrigido-.pdf

7. Abebe AM, Kassaw MW, Mengistu FA. Assessment of Factors Affecting the Implementation of Integrated Management of Neonatal and Childhood Illness for Treatment of under Five Children by Health Professional in Health Care Facilities in Yifat Cluster in North Shewa Zone, Amhara Region, Ethiopia. Int J Pediatr. 2019;2019:9474612. http://dx.doi.org/10.1155/2019/9474612. PMid:31949443.

8. Portaria no 1.130, de 5 de agosto de 2015 (BR). Institui a Política Nacional de Saúde da Criança (PNAISC) no âmbito do Sistema Único de Saúde (SUS). Diário Oficial da União [periódico na internet], Brasília (DF) 2015 [citado 2018 jun 21]. Disponível em: http://bvsms.saude.gov. br/bvs/saudelegis/gm/2015/prt1130_05_08_2015.html

9. Conselho Nacional de Secretários de Saúde (BR). A Atenção Primária e as Redes de Atenção à Saúde [Internet]. Brasília, DF: CONASS;2015 [citado 2018 abr 14]. Disponível em: http://www.conass.org.br/biblioteca/ pdf/A-Atencao-Primaria-e-as-Redes-de-Atencao-a-Saude.pdf

10. Zaslavsky R, Goulart BN. Migração pendular e atenção à saúde na região de fronteira. Cien Saude Colet. 2017;22(12):3981-6. http://dx.doi. org/10.1590/1413-812320172212.03522016. PMid:29267715.

11. de Mello F, Victora CG, Gonçalves H. Saúde nas fronteiras: análise quantitativa e qualitativa da clientela do centro materno infantil de Foz do Iguaçu, Brasil. Cien Saude Colet. 2015;20(7):2135-45. http://dx.doi. org/10.1590/1413-81232015207.09462014. PMid:26132253.

12. Minayo MCS. O desafio do conhecimento. Pesquisa qualitativa em saúde. 14. ed. São Paulo: Hucitec; 2014. 406 p.

13. Perry R, Leach V, Penfold C, Davies P. An overview of systematic reviews of complementary and alternative therapies for infantile colic. Syst Rev. 2019;8(1):271. http://dx.doi.org/10.1186/s13643-019-1191-5.PMid:31711532.

14. Tarciuc P, Stanescu AMA, Diaconu CC, Paduraru L, Duduciuc A, Diaconescu S. Patterns and Factors Associated with Self-Medication among the Pediatric Population in Romania. Medicina (Kaunas). 2020;56(6):312. http://dx.doi.org/10.3390/medicina56060312. PMid:32630388.

15. Lima MRA, Nunes MLA, Kluppel BLP, Medeiros SM, Sa LD. Nurses' performance on indigenous and African-Brazilian healthcare practices. Rev Bras Enferm. 2016;69(5):788-94. http://dx.doi.org/10.1590/00347167.2016690504. PMid:27783725.
16. Hirano AR, Baggio MA, Ferrari RP. Amamentação, alimentação complementar e segurança alimentar em uma região de fronteira. CogitareEnferm. 2021;26:e72739. http://dx.doi.org/10.5380/ce.v26i0.72739.

17. Da Silva JG, Gomes GC, Costa AR, Juliano LF, Arruda CP, De Carvalho LN. A prática da automedicação em crianças por seus pais: atuação da enfermagem. Rev Enferm UFPE. 2018;12(6):1570-7. http://dx.doi. org/10.5205/1981-8963-v12i6a230779p1570-1577-2018.

18. Konder MT, O'Dwyer G. A integração das Unidades de Pronto Atendimento (UPA) com a rede assistencial no município do Rio de Janeiro, Brasil. Interface (Botucatu). 2016;20(59):879-92. http://dx.doi org/10.1590/1807-57622015.0519.

19. Vaz EMC, Collet N, Cursino EG, Forte FDS, Magalhães RKBP, Reichert APS. Care coordination in Health Care for the child/adolescent in chronic condition. Rev Bras Enferm. 2018;71(suppl. 6):2612-9. https:// doi.org/10.1590/0034-7167-2017-0787.

20. Secretaria de Estado da Saúde do Paraná (PR). Superintendência de Atenção à Saúde. Linha Guia da Rede Mãe Paranaense. $7^{a}$ ed [Internet] Curitiba; 2018 [citado 2020 dez 20]. Disponível em: http://www.saude. pr.gov.br/arquivos/File/LinhaGuiaMaeParanaense_2018.pdf

21. Seid SS, Sendo EG. A survey on Integrated Management of Neonatal and Childhood Illness implementation by nurses in four districts of West Arsi zone of Ethiopia. Pediatric Health Med Ther. 2018;9:1-7. http:// dx.doi.org/10.2147/PHMT.S144098. PMid:29443325.

22. Aikes S, Rizzotto MLF. Integração regional em cidades gêmeas do Paraná, Brasil, no âmbito da saúde. Cad Saude Publica. 2018;34(8):e00182117. http://dx.doi.org/10.1590/0102-311x00182117. PMid:30133667.

23. Nascimento VA, Andrade SMO. As armas dos fracos: estratégias, táticas e repercussões identitárias na dinâmica do acesso à saúde na fronteira Brasil/Paraguai. Horiz Antropol. 2018;24(50):181-214. http:// dx.doi.org/10.1590/s0104-71832018000100007.

24. Mello DF, Silva RMM, Pancieri L. Êxito técnico e sucesso em visita domiciliar para o cuidado da saúde da criança. Rev Pesquisa Qualitativa [Internet]. 2017;5(7):13-22 [citado 2020 dez 20]. Disponível em: https:// editora.sepq.org.br/index.php/rpq/article/view/46/60

25. Mochizuke KC. Influência do atendimentoemsaúde à estrangeirosemumacidadefronteiriçabrasileira. J Health NPEPS. [Internet]. 2017;2(1) [citado 2020 dez 20]. Disponível em: https:// periodicos.unemat.br/index.php/jhnpeps/article/view/1824/1674

26. Gomide MFS, Pinto IC, Bulgarelli AF, Santos ALP, Serrano Gallardo MP. A satisfação do usuário com a atenção primária à saúde: uma análise do acesso e acolhimento. Interface Comunicacao Saude Educ. 2017 set 21;22(65):387-98. http://dx.doi.org/10.1590/1807-57622016.0633.

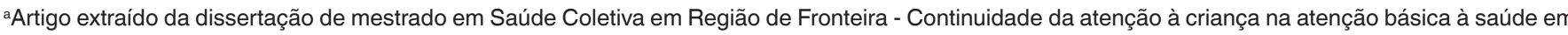
região de fronteira - de Taigra Morgana Picco, orientada pela Dra. Maria Aparecida Baggio. Universidade Estadual do Oeste do Paraná. Ano 2019.
} 